\title{
ON SPECIAL $W$-SURFACES
}

\section{SHIING-SHEN CHERN}

A $W$-surface is a surface in ordinary Euclidean space for which there is a functional relation between the principal curvatures $k_{1}, k_{2}$ :

$$
W\left(k_{1}, k_{2}\right)=0 .
$$

We shall be interested in those $W$-surfaces for which (1) can be written in the form

$$
f(H, \mu)=0, \quad \mu=H^{2}-K,
$$

where $H$ and $K$ are the mean curvature and the Gaussian curvature respectively and where $f$ is of class $C^{1}$. For such $W$-surfaces we have

$$
\begin{aligned}
& \partial W / \partial k_{1}=f_{H} / 2+\left(k_{1}-k_{2}\right) f_{\mu} / 2, \\
& \partial W / \partial k_{2}=f_{H} / 2-\left(k_{1}-k_{2}\right) f_{\mu} / 2 .
\end{aligned}
$$

It follows that at an umbilic $\left(k_{1}=k_{2}\right)$ we have

$$
\partial W / \partial k_{1} \cdot \partial W / \partial k_{2}=f_{H}^{2} / 4 \geqq 0 .
$$

A $W$-surface (2) is called special if $f_{H} \neq 0$ at every umbilic.

Apparently there are very few closed special $W$-surfaces. ${ }^{1}$ The following theorem is due to H. Hopf : ${ }^{2}$

The only closed special analytic $W$-surfaces of genus zero are spheres.

It was P. Hartman and A. Wintner ${ }^{3}$ who succeeded in removing the analyticity assumption in Hopf's Theorem. Their theorem can be stated as follows:

Let $S$ be a closed special $W$-surface of genus zero, which is $C^{3}$-imbedded in Euclidean space. Then $S$ is a sphere.

We shall show that the formalism developed in the preceding paper gives a very simple proof of the theorem of Hartman-Wintner. ${ }^{4}$

Received by the editors November 15, 1954.

1 The notion of a special $W$-surface was first introduced by the author in the paper Some new characterizations of the Euclidean sphere, Duke Math. J. vol. 12 (1945) pp. 279-290. I take this opportunity to mention that the proof of Theorem 3 in that paper is not valid. So far as I know, the question whether there exist closed surfaces of constant mean curvature and of genus $>0$ remains unanswered.

${ }^{2} \mathrm{H}$. Hopf, Über Flächen mit einer Relation zwischen den Hauptkrümmungen, Mathematische Nachrichten vol. 4 (1950-1951) pp. 232-249.

${ }^{3} \mathrm{P}$. Hartman and A. Wintner, Umbilical points and W-surfaces, Amer. J. Math. vol. 76 (1954) pp. 502-508.

4 The hypotheses of Hartman-Wintner are weakened in one respect in that the function $f$ in (2) is here assumed to be of class $C^{1}$, while they supposed $f$ to be of class $C^{2}$. 
Let $x, y$ be isothermal parameters, and let $z=x+i y$. Let $E\left(d x^{2}\right.$ $\left.+d y^{2}\right), L d x^{2}+2 M d x d y+N d y^{2}$ be respectively the first and second fundamental forms of the surface, so that

$$
\begin{aligned}
& 2 E H=L+N, \\
& E^{2} K=L N-M^{2} .
\end{aligned}
$$

Put

$$
w=(L-N) / 2-i M .
$$

Then Codazzi's equations can be written

$$
w_{\mathbf{z}}=E H_{\mathbf{z}} \text {. }
$$

Also we have from (5)

$$
\mu=H^{2}-K=w \bar{w} / E^{2} .
$$

If the surface is a special $W$-surface satisfying (2), we have, in a neighborhood of an umbilic,

$$
H_{2}=-\left(f_{\mu} / f_{H}\right) \mu_{z} .
$$

This means that $w$ satisfies a nonlinear differential equation of the form

$$
w_{\bar{z}}=P(w \bar{w})_{z}+Q w \bar{w},
$$

where

$$
P=-f_{\mu} / E f_{H}, \quad Q=2 E_{z} / E^{2} \cdot f_{\mu} / f_{H} .
$$

Following the procedure of Hartman-Wintner, the proof of their theorem depends on the lemmas:

LEMMA 1. Let $w(z, \bar{z})$ be a solution of (9), in a sufficiently small neighborhood of $z=0$, at which $w=0$. Then $\lim _{z \rightarrow 0} w(z, \bar{z}) z^{-k}$ exists if $w=o\left(|z|^{k-1}\right)$.

LEMMA 2. Under the hypotheses of Lemma 1, suppose that $w=o\left(|z|^{k-1}\right)$ for all $k$. Then $w(z, \bar{z}) \equiv 0$ in a neighborhood of $z=0$.

From these lemmas we derive immediately the theorem of Hartman-Wintner. In fact, it follows from Lemma 2 that if $0(z=0)$ does not have a neighborhood which consists entirely of umbilics, there exists an integer $k$, such that $w=o\left(|z|^{k-1}\right), w \neq o\left(|z|^{k}\right)$. By Lemma 1, $\lim _{z \rightarrow 0} w(z, \bar{z}) z^{-k}$ exists and is $\neq 0$. We can therefore write

$$
w(z, \bar{z})=c z^{k}+o\left(|z|^{k}\right)
$$$$
c \neq 0 \text {. }
$$ 
It follows that the umbilic 0 is isolated and has an index $-k<0$. By well-known arguments this implies the theorem of HartmanWintner.

It remains to prove the above lemmas. For this purpose let $D$ be a disc of radius $R$ about 0 , and $C$ its boundary circle. There exists a constant $A>0$, such that in $D$,

$$
\left|P(w \bar{w})_{z}+Q w \bar{w}\right| \leqq A|w| .
$$

Suppose that $w=o\left(|z|^{k-1}\right)$. Let $\zeta=\xi+i \eta$ be an interior point of $D$. Then we have, for $\zeta \neq 0$,

$$
d\left\{\frac{w d z}{z^{k}(z-\zeta)}\right\}=\frac{P(w \bar{w})_{z}+Q w \bar{w}}{z^{k}(z-\zeta)} d \bar{z} \wedge d z .
$$

Application of Stokes Theorem gives

$$
-2 \pi i w(\zeta, \bar{\zeta}) \zeta^{-k}+\int_{C} \frac{w d z}{z^{k}(z-\zeta)}=\iint_{D} \frac{P(w \bar{w})_{z}+Q w \bar{w}}{z^{k}(z-\zeta)} d \bar{z} d z
$$

It follows that

$$
\begin{aligned}
2 \pi\left|w(\zeta, \bar{\zeta}) \zeta^{k}\right| \leqq & \int_{C}\left|\frac{w(z, \bar{z})}{z^{k}(z-\zeta)}\right||d z| \\
& +2 A \iint_{D}\left|\frac{w(z, \bar{z})}{z^{k}(z-\zeta)}\right| d x d y .
\end{aligned}
$$

We multiply this inequality by $d \xi d \eta /\left|\zeta-z_{0}\right|, z_{0} \in D$, and integrate over $D$. Remembering that

$$
\begin{gathered}
\iint_{D} \frac{d x d y}{|z-\zeta|}<2 R, \\
\frac{1}{\left|(z-\zeta)\left(z_{0}-\zeta\right)\right|}=\frac{1}{\left|z-z_{0}\right|}\left|\frac{1}{z-\zeta}+\frac{1}{\zeta-z_{0}}\right|,
\end{gathered}
$$

we get from this integration

$$
\begin{aligned}
2 \pi \int_{D}\left|\frac{w(z, \bar{z})}{z^{k}(z-\zeta)}\right| d x d y \leqq & 4 R \int_{C}\left|\frac{w(z, \bar{z})}{z^{k}(z-\zeta)}\right||d z| \\
& +8 A R \iint_{D}\left|\frac{w(z, \bar{z})}{z^{k}(z-\zeta)}\right| d x d y
\end{aligned}
$$




$$
(2 \pi-8 A R) \iint_{D}\left|\frac{w(z, \bar{z})}{z^{k}(z-\zeta)}\right| d x d y \leqq 4 R \int_{C}\left|\frac{w(z, \bar{z})}{z^{k}(z-\zeta)}\right||d z|
$$

We choose $R$ so small that $2 \pi-8 A R>0$. Then

$$
\iint_{D}\left|w(z, \bar{z}) / z^{k}(z-\zeta)\right| d x d y
$$

is bounded as $\zeta \rightarrow 0$, and the same is true of $\left|w(\zeta, \bar{\zeta}) \zeta^{-k}\right|$. It follows that $\left|(w \bar{w})_{\zeta} \zeta^{-k}\right|$ is bounded and from (14) that $\lim _{\zeta \rightarrow 0} w(\zeta, \bar{\zeta}) \zeta^{-k}$ exists. This proves Lemma 1.

To prove Lemma 2 we multiply (15) by $d \xi d \eta$ and integrate over $D$. This gives

$$
\begin{aligned}
2 \pi \int_{D}\left|w(z, \bar{z}) z^{-k}\right| d x d y \leqq & 2 R \int_{C}\left|w(z, \bar{z}) z^{-k}\right||d z| \\
& +4 A R \iint_{D}\left|w z^{-k}\right| d x d y
\end{aligned}
$$

or

$$
(2 \pi-4 A R) \iint_{D}\left|w(z, \bar{z}) z^{-k}\right| d x d y \leqq 2 R \int_{C}\left|w(z, \bar{z}) z^{-k}\right||d z| .
$$

Suppose there exists a $z_{0}$ such that $w\left(z_{0}, \bar{z}_{0}\right) \neq 0,\left|z_{0}\right|<R$. Then the left-hand side of the above inequality is $\geqq a\left|z_{0}\right|^{-k}$, and the right-hand side is $\leqq b R^{-k}$, where $a$ and $b$ are positive constants independent of $k$. The hypothesis of Lemma 2 implies that $\left|z_{0} / R\right|^{k} \geqq a / b$ for all $k$, which is a contradiction. It follows that $w(z, \bar{z})$ vanishes identically for $|z|<R$.

INSTITUTE for Advanced STUdy AND

UNIVERSITY OF CHICAGO 